\title{
Vorinostat enhances gefitinib-induced cell death through reactive oxygen species-dependent cleavage of HSP90 and its clients in non-small cell lung cancer with the EGFR mutation
}

\author{
SANG EUN PARK ${ }^{1}$, DONG EUN KIM ${ }^{1}$, MI JOUNG KIM ${ }^{1}$, JEE SUK LEE ${ }^{1}$, JIN KYUNG RHO $^{1,2}$, \\ SEONG-YUN JEONG ${ }^{1,2}$, EUN KYUNG CHOI ${ }^{3}$, CHOUNG-SOO KIM ${ }^{4}$ and JUNG JIN HWANG ${ }^{1,2}$ \\ ${ }^{1}$ Asan Institute for Life Sciences, Asan Medical Center; Departments of ${ }^{2}$ Convergence Medicine, \\ ${ }^{3}$ Radiation Oncology, and ${ }^{4}$ Urology, Asan Medical Center, University of Ulsan College of Medicine, \\ Seoul 05505, Republic of Korea
}

Received March 30, 2018; Accepted September 20, 2018

DOI: $10.3892 /$ or.2018.6814

\begin{abstract}
Although different mechanisms of acquired resistance to epidermal growth factor receptor (EGFR)-tyrosine kinase inhibitors (TKIs) have been reported in non-small cell lung cancers (NSCLCs), the optimal treatment for patients with acquired resistance has not been clearly defined. The purpose of this study was to investigate the antitumor effects of gefitinib in combination with vorinostat, a potent histone deacetylase inhibitor (HDACI), and their associated molecular mechanisms in relation to activating apoptosis in NSCLC. The treatment using a combination of vorinostat and gefitinib was more potent in promoting cell death by activating apoptosis than gefitinib alone in parental PC9 cells that harbor an EGFR-activating mutation (EGFR exon 19 deletion) and gefitinib-resistant PC9 cells (PC9GR) with an EGFR T790M mutation. This combination induced heat shock protein 90 (HSP90) cleavage and reduced the level of HSP90 client proteins, including EGFR, MET and AKT, in PC9 and PC9GR cells. The addition of 4-(2-aminoethyl) benzenesulfonyl fluoride hydrochloride, a scavenger of reactive oxygen species (ROS), inhibited the degradation of HSP90 client proteins and HSP90 cleavage that was induced by co-treatment as well as the cleavage of caspase- 3 , caspase- 8 , and caspase- 9
\end{abstract}

Correspondence to: Professor Jung Jin Hwang, Department of Convergence Medicine, Asan Medical Center, University of Ulsan College of Medicine, 88 Olympic-ro 43-gil, Songpa-gu, Seoul 05505, Republic of Korea

E-mail: jjhwang@amc.seoul.kr

Professor Choung-Soo Kim, Department of Urology, Asan Medical Center, University of Ulsan College of Medicine, 88 Olympic-ro 43-gil, Songpa-gu, Seoul 05505, Republic of Korea

E-mail: cskim@amc.seoul.kr

Key words: epidermal growth factor receptor, gefitinib, histone deacetylase inhibitor, heat shock protein 90, non-small cell lung cancer, reactive oxygen species and cell death. We also observed that cleavage of HSP90 and its clients were blocked when caspases were inhibited. These results revealed that co-treatment with vorinostat and gefitinib induced ROS-dependent caspase activation, leading to the downregulation of HSP90 clients through HSP90 cleavage. Collectively, our findings provide a new basis for strategies that combine vorinostat with an EGFR-TKI to reverse EGFR-TKI resistance in NSCLC.

\section{Introduction}

First-generation epidermal growth factor receptor (EGFR)tyrosine kinase inhibitors (TKIs), gefitinib and erotinib, are used to treat patients with advanced non-small cell lung cancer (NSCLC) that harbors an EGFR-activating mutation, especially a deletion in exon 19 and an L858R mutation in exon 21. However, despite their initial response to EGFR-TKI treatment, most patients eventually develop resistance and consequently relapse. The mechanisms by which resistance to EGFR-TKIs is acquired include a mutation of EGFR T790M within exon 20. Although EGFR T790M-specific EGFR-TKIs have been developed, resistance also occurs with this class of EGFR-TKIs. The distinct mechanisms by which resistance to EGFR-TKIs is acquired are the activation of bypass signaling (MET, AXL and/or ERBb2) and their downstream pathways (PI3K, AKT and/or MEK). Therefore, new strategies to overcome multifactorial resistance to EGFR-TKIs are needed to improve the efficacy of this treatment.

Heat shock protein 90 (HSP90), which is highly conserved evolutionarily and ubiquitously expressed, has been attributed to the folding, stabilization, and proteolytic degradation of client oncoproteins involved in the proliferation of tumors (1). Inhibition of HSP90 results in degradation of its clients, including receptor tyrosine kinases (RTKs), and its downstream signaling molecules, such as AKT, MEK and Src (2). Therefore, HSP90 represents an engaging molecular target for anticancer therapy and has been under preclinical and clinical development for the treatment of NSCLC (3). Although several HSP90 inhibitors have shown promising results in preclinical research and 17-AAG, the first HSP90 inhibitor, has entered 
into a phase I clinical trial, this drug might not be approved to treat lung cancer because of its poor drug-like properties, such as poor solubility, poor patient enrichment, suboptimal target inhibition, and off-target toxicities (4).

The function of HSP90 is regulated by post-translational modification. Acetylation of HSP90 has been observed in cancer cells treated with histone deacetylase inhibitors (HDACIs), leading to destabilization of its client proteins. First, HDACIs emerged as potential multifunctional agents that regulate chromatin remodeling and are crucial to the epigenetic regulation of various genes, such as tumor suppressors and oncogenes. Later, it was discovered that HDAC6 is located in the cytosol and modulates the acetylation of cytosolic proteins, including HSP90, p53 and tubulin. Recent data suggest that HDACIs can increase sensitivity and reverse resistance to EGFR-TKIs in lung cancer cells by inducing E-cadherin expression in those cells (5). Thus, ongoing research on incorporating HDACIs into NSCLC treatment concentrates on combining HDACIs with EGFR-TKIs (6). Vorinostat, also called suberoylanilide hydroxamic acid (SAHA), is an inhibitor of class I and II histone deacetylases that regulate the transcription of genes involved in cell survival and apoptosis and has demonstrated considerable antigrowth effects on NSCLC cells (7). Given the potential synergy between HDACIs and EGFR-TKIs, we conducted a study in which gefitinib and vorinostat were combined to target HSP90 in NSCLC with an EGFR mutation.

\section{Materials and methods}

Cell culture and reagents. The human NSCLC cell line PC9 and gefitinib-resistant PC9 (PC9GR) cells were provided by Dr Rho (Asan Medical Center, Seoul, Korea) (8,9). All cell lines were cultured in Gibco ${ }^{\circledR}$ RPMI-1640 medium (Thermo Fisher Scientific,Inc., Waltham,MA,USA) containing 10\% fetal bovine serum (FBS; Gibco; Thermo Fisher Scientific, Inc.) and $1 \%$ penicillin/streptomycin (Gibco; Thermo Fisher Scientific, Inc.) at $37^{\circ} \mathrm{C}$ in an atmosphere containing $5 \% \mathrm{CO}_{2}$. The following compounds were used in this study: Gefitinib (Iressa, AstraZeneca, London, UK); vorinostat (Crystal Genomics, Inc., Seoul, Korea); 4-(2-aminoethyl)-benzenesulfonyl fluoride hydrochloride (AEBSF, Sigma-Aldrich; Merck KGaA, Darmstadt, Germany); and Z-VAD-FMK, Z-LEHD-FMK, Z-DEVD-FMK and Z-IETD-FMK (R\&D Systems, Minneapolis, MN, USA). All reagents were purchased from Sigma-Aldrich (Merck KGaA, Darmstadt, Germany) unless otherwise specified.

Drug treatment. The cells were seeded at a density of $3 \times 10^{3}$ cells/well in 96-well plates. After overnight incubation, cells were pretreated with AEBSF, Z-VAD, Z-IETD or Z-LEHD for $2 \mathrm{~h}$, followed by treatment with gefitinib and/or vorinostat in RPMI-1640 medium containing 10\% FBS.

Cell viability assay and combination index analysis. The percentage of viable cells was determined using the CellTiter-Glo luminescent cell viability assay according to the manufacturer's recommendations (Promega Corp., Madison, WI, USA). The combination index (CI) was calculated using with CalcuSyn v. 2.1.1 (BioSoft, Cambridge, UK), which is based on the Chou-Talalay method and provides qualitative information on drug interaction. Antagonism is defined as $\mathrm{CI}>1.0$, an additive effect is $\mathrm{CI}=1.0$, and synergism is $\mathrm{CI}<1.0$. After achieving a maximum effect from the drugs tested on cancer cells, a mean CI was obtained from the value of the fraction of cell growth that is affected (Fa) (e.g., $\mathrm{Fa}=0.5$ is equivalent to a $50 \%$ reduction in cell growth).

Western blot analysis. Cell lysates were prepared from $1 \times 10^{7}$ cells by dissolving cell pellets in $200 \mu 1$ lysis buffer containing $20 \mathrm{mM}$ Tris- $\mathrm{HCl}$ (pH 7.4), $150 \mathrm{mM} \mathrm{NaCl}, 1 \%$ (v/v) Nonidet P-40, 0.5\% (w/v) sodium deoxycholate, $0.1 \%(\mathrm{w} / \mathrm{v})$ sodium dodecyl sulfate, and a protease inhibitor cocktail (Roche Diagnostics, Mannheim, Germany). Cell lysates were centrifuged at $20,000 \mathrm{x}$ g for $20 \mathrm{~min}$, and the protein concentrations were determined using a BCA Protein Assay kit (Pierce, Rockford, IL, USA). Lysates with sodium dodecyl sulfate-polyacrylamide gel electrophoresis (SDS-PAGE) sample buffer were heated for $5 \mathrm{~min}$ at $100^{\circ} \mathrm{C}$ and $50 \mu \mathrm{l}$ proteins were resolved using SDS-PAGE 6-10\% gels. The gels were transferred to polyvinylidene difluoride (PVDF) membranes (Millipore, Bedford, MA, USA) and the blots were probed with specific antibodies. Primary antibodies against the various proteins were obtained from the following sources: Mcl-1 (cat. no. sc-819) and GAPDH (cat. no. sc-20357) were obtained from Santa Cruz Biotechnology (Dallas, TX, USA) and HSP90 (cat. no. 4874), caspase-3 (cat. no. 9662), caspase-8 (cat. no. 9746), caspase-9 (cat. no. 9502), PARP (cat. no. 9542), Bax (cat. no. 2772), HER2/ErbB2 (cat. no. 2242), pEGFR (Y1068) (cat. no. 2234), EGFR (cat. no. 2646), pMET (T1234/1235) (cat. no. 3129), MET (cat. no. 4560), pAKT (S473) (cat. no. 9271) and AKT (cat. no. 9272) were obtained from Cell Signaling Technology (Danvers, MA, USA). The dilution ratio of these antibodies was 1:1,000. Secondary antibodies purchased from as follows: goat anti-mouse IgG-horseradish peroxidase (HRP) (1:5,000; cat. no. 32430), goat anti-rabbit IgG-HRP (1:5,000; cat. no. 32460) obtained from Pierce Biotechnology (Rockford, IL, USA) and mouse anti-goat IgG-HRP (1:5,000; cat. no. sc-2354) were obtained from Santa Cruz Biotechnology. The membranes were developed using Immobilon Western ECL solution (Millipore) and detected using the Kodak Image Station 4000MM system (Kodak, Rochester, NY, USA). The blots were analyzed using Kodak Molecular Imaging, version 4.0.5 software (Eastman Kodak Company, Rochester, NY, USA).

Labeling of the cell nuclei with Hoechst 33258 to detect nuclear fragmentation. After treatment, the cells were fixed with $4 \%$ paraformaldehyde for $10 \mathrm{~min}$ at room temperature and washed twice with phosphate-buffered saline (PBS). The nuclei were then labeled with $2.5 \mu \mathrm{g} / \mathrm{ml}$ Hoechst 33258 for $15 \mathrm{~min}$ and washed again three times with PBS. Fluorescent micrographs of the labeled nuclei were captured using an Olympus IX71 fluorescence microscope (Olympus Corp., Tokyo, Japan). Five images per well were captured and the fragmented nuclei were counted.

Flow cytometry. After treatment, apoptosis was measured using the FITC Annexin V apoptosis detection kit II (BD Pharmingen; BD Biosciences, San Diego, CA, USA) in accordance with the manufacturer's instructions. The stained 
cells were detected using a BD FACSCanto 2 flow cytometer and analyzed by BD FACSDiva (version 8.0; BD Biosciences).

Assessment of reactive oxygen species production. After the drug-containing medium was removed, the cells were washed with serum-free medium and incubated with $10 \mu \mathrm{M}$ 2',7'-dichlorodihydrofluorescin diacetate $\left(\mathrm{H}_{2} \mathrm{DCF}-\mathrm{DA}\right.$, Invitrogen; Thermo Fisher Scientific, Inc.) for $30 \mathrm{~min}$ in the dark. Levels of reactive oxygen species (ROS) were continuously monitored for up to $20 \mathrm{~min}$. The fluorescence images were obtained using an Olympus IX71 fluorescence microscope (Olympus Corp).

Statistical analyses. SigmaPlot version 13.0 (Systat Software, Inc., Erkrath, Germany) was used to analyze the significance of all results. Statistical significance was determined by one-way analysis of variance using (ANOVA) $(\mathrm{P}<0.05)$. A post-hoc test of ANOVA was conducted by performing a Turkey's test. All experiments were performed in triplicate, and all data are expressed as the mean \pm standard deviation.

\section{Results}

Co-treatment with gefitinib and vorinostat potentiates apoptotic cell death in lung cancer. First, we examined the inhibitory effect of the HDACI vorinostat on the viability of NSCLC PC9 and gefitinib-resistant PC9GR cells using a CellTiter-Glo assay. The PC9 cell line has a deletional mutation in EGFR exon 19 and the PC9GR cell line is resistant to gefitinib by having acquired a secondary T790M mutation in EGFR exon $20(8,9)$. Exposure to vorinostat efficiently reduced the viability of both cell lines in a concentration-dependent manner (Fig. 1A). The $\mathrm{IC}_{50}$ values of vorinostat were 2.661 and $6.282 \mu \mathrm{M}$ for PC9GR and PC9 cells, respectively. As shown in Fig. 1B, vorinostat significantly decreased the level of total and phosphorylated EGFR and MET, which are responsible for the survival of lung cancer cells $(10,11)$.

Next, we examined the effect of vorinostat on gefitinib sensitivity in PC9 and PC9GR cells. In accordance with previous reports $(8,9)$, treatment with $0.01 \mu \mathrm{M}$ gefitinib alone significantly decreased the viability of PC9 cells, but it had no effect on PC9GR cells (Fig. 2A, upper graphs). Notably, co-treatment with gefitinib and vorinostat resulted in a synergistic effect in a concentration-dependent manner not only on PC9 but also on PC9GR cells. The CI values were $<1.0$ in all combinations, representing synergistic interaction, except for that following treatment with $10 \mu \mathrm{M}$ vorinostat on PC9GR cells (Fig. 2A, lower tables). To determine the types of cell death induced by the combined treatment, we examined the changes in apoptosis-related proteins. Co-treatment with gefitinib and vorinostat induced cleavage of caspase- 3 and PARP and increased the expression of Bax, a pro-apoptotic Bcl-2 family protein, in PC9 and PC9GR cells (Fig. 2B). Mcl-1, an anti-apoptotic Bcl-2 family protein, decreased in both cell lines after combined treatment. In accordance with these results, we also observed nuclear fragmentation and Annexin V/propidium iodide (PI)-positive cells in both cell lines exposed to the combined treatment and the reverse of these events by adding the pan-caspase inhibitor Z-VAD (Fig. 2C and D). These findings indicate that apoptosis mediates the substantial
A

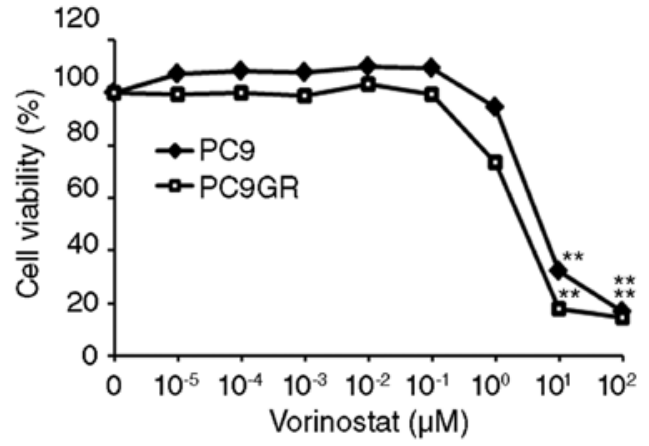

$\mathbf{B}$

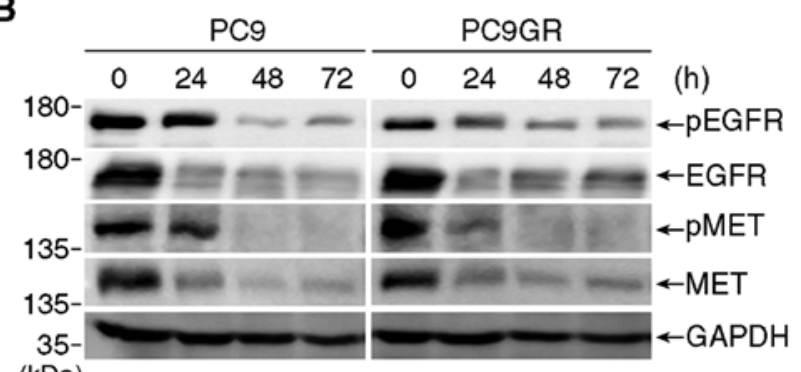

$(\mathrm{kDa})$

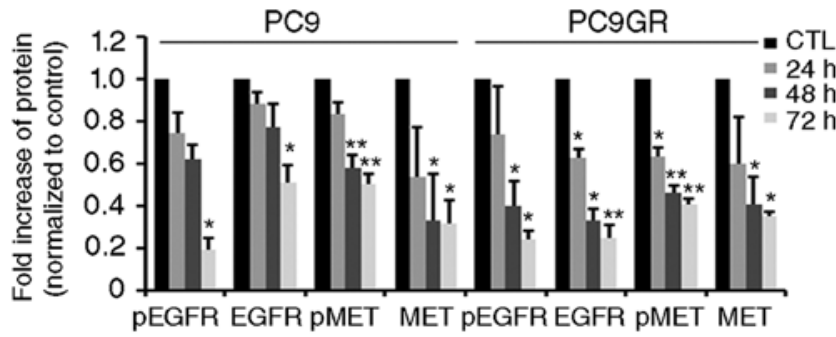

Figure 1. Vorinostat inhibits cell growth and decreases epidermal growth factor receptor (EGFR) and MET proteins in PC9 and gefitinib-resistant PC9 (PC9GR) cells. (A) PC9 and PC9GR cells were treated with the indicated concentrations of vorinostat for $48 \mathrm{~h}$, and the percentage of viable cells was measured using CellTiter-Glo luminescent cell viability assay. Data are expressed as the mean $\pm \mathrm{SD}\left(\mathrm{n}=3,{ }^{* *} \mathrm{P}<0.001\right)$. (B) Cells were treated with $10 \mu \mathrm{M}$ vorinostat for the indicated times and the levels of EGFR, MET, pEGFR, and pMET were detected by western blotting. Protein levels were analyzed by densitometry $\left(\mathrm{n}=3,{ }^{*} \mathrm{P}<0.05,{ }^{* *} \mathrm{P}<0.001\right)$.

synergistic effects of co-treatment with gefitinib and vorinostat in NSCLC cells with EGFR-activating mutations, including exon 19 deletion and T790M mutation.

Co-treatment leads to HSP90 cleavage and degradation of HSP9O client proteins in an ROS-dependent manner. To identify the underlying mechanisms involved in apoptosis, we examined the levels of EGFR and MET proteins and their downstream target, AKT. As shown in Fig. 3A, co-treatment with gefitinib and vorinostat significantly decreased the levels of total EGFR, MET and AKT proteins and their phosphorylation compared with levels following treatment with each drug alone. Since EGFR, MET and AKT are the clients of HSP90 (12), we examined the level of HSP90 proteins. Treatment with vorinostat alone increased HSP90 cleavage, and co-treatment significantly increased HSP90 cleavage in both PC9 and PC9GR cells (Fig. 3B).

It has been reported that oxidative stress caused by various anticancer drugs induce HSP90 cleavage and degrade its client proteins in cancer cells $(13,14)$. In accordance with these reports, we observed a synergistic increase in ROS by co-treatment 
A

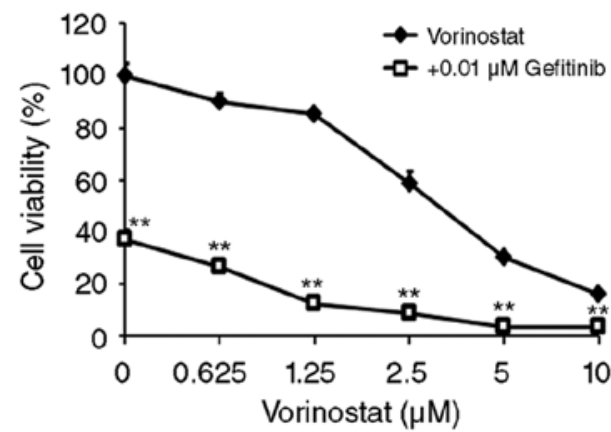

PC9GR

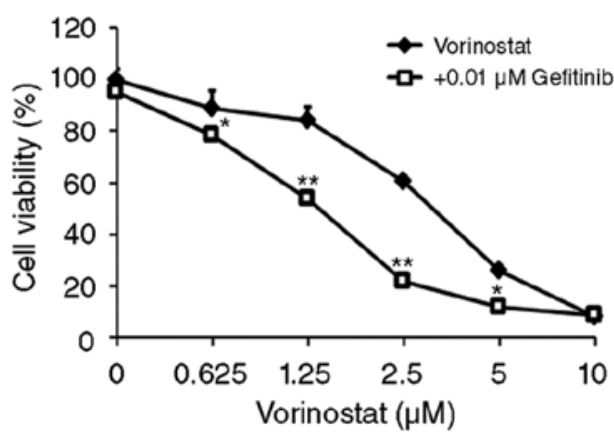

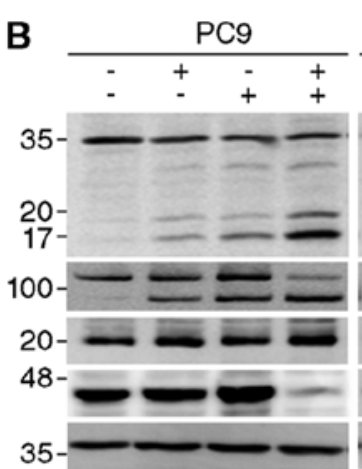
(kDa)

\begin{tabular}{|c|c|c|c|}
\hline Gefitinib $(\mu \mathrm{M})$ & Vorinostat $(\mu \mathrm{M})$ & $\mathrm{Fa}$ & $\mathrm{Cl}$ \\
\hline \multirow{4}{*}{0.01} & 0.625 & 0.735 & 0.576 \\
\cline { 2 - 4 } & 1.25 & 0.874 & 0.286 \\
\cline { 2 - 4 } & 2.5 & 0.913 & 0.383 \\
\cline { 2 - 4 } & 5 & 0.961 & 0.266 \\
\cline { 2 - 4 } & 10 & 0.962 & 0.350 \\
\hline
\end{tabular}

\begin{tabular}{|c|c|c|c|}
\hline Gefitinib $(\mu \mathrm{M})$ & Vorinostat $(\mu \mathrm{M})$ & $\mathrm{Fa}$ & $\mathrm{Cl}$ \\
\hline \multirow{4}{*}{0.01} & 0.625 & 0.215 & 0.348 \\
\cline { 2 - 4 } & 1.25 & 0.463 & 0.375 \\
\cline { 2 - 4 } & 2.5 & 0.779 & 0.442 \\
\cline { 2 - 4 } & 5 & 0.879 & 0.618 \\
\cline { 2 - 4 } & 10 & 0.911 & 1.034 \\
\hline
\end{tabular}

C
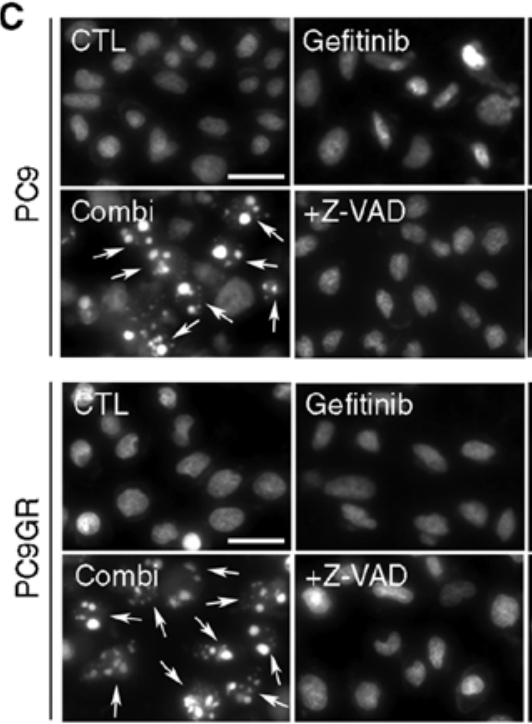

PC9GR
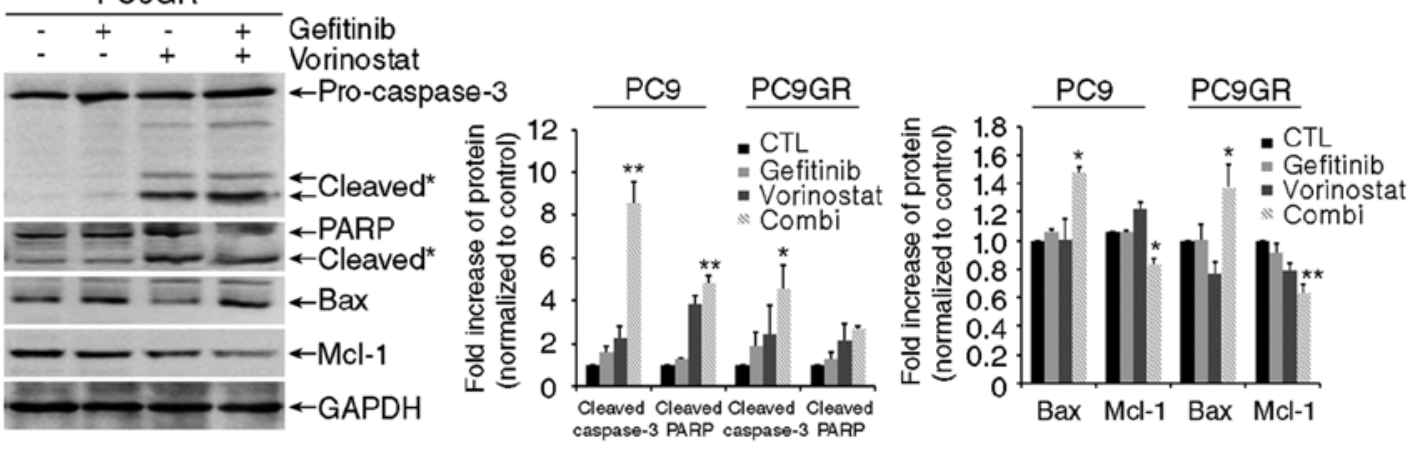

Figure 2. Co-treatment with gefitinib and vorinostat increases apoptosis. (A) PC9 and PC9GR cells were treated with $0.01 \mu \mathrm{M}$ gefitinib and/or the indicated concentrations of vorinostat for $48 \mathrm{~h}$, and cell viability was assessed. Data represent mean $\pm \mathrm{SD}\left(\mathrm{n}=3,{ }^{*} \mathrm{P}<0.05,{ }^{* *} \mathrm{P}<0.001\right)$. The fraction affected value $(\mathrm{Fa})$, indicating the fraction of cells inhibited after drug exposure, and the combination index (CI) were calculated using CalcuSyn. (B) Cells were treated with $0.01 \mu \mathrm{M}$ gefitinib and/or $5 \mu \mathrm{M}$ vorinostat for $48 \mathrm{~h}$, and levels of cleaved capsase-3, PARP, Bax and Mcl-1 were determined by western blotting. GAPDH was used as a loading control. Cleavage products are labeled with an asterisk $\left(\mathrm{n}=3,{ }^{*} \mathrm{P}<0.05,{ }^{* *} \mathrm{P}<0.001\right)$. (C) Nuclear staining with $10 \mu \mathrm{M}$ Hoechst 33342 in cells treated with $0.01 \mu \mathrm{M}$ gefitinib and/or $5 \mu \mathrm{M}$ vorinostat in the presence or absence of $50 \mathrm{M}$ Z-VAD-FMK for $48 \mathrm{~h}$. Left panels: Hoechst staining. Scale bar, $50 \mu \mathrm{m}$. Right graphs: quantification of fragmented nuclei [mean $\pm \mathrm{SD}, \mathrm{n}=3 ;{ }^{*} \mathrm{P}<0.05,{ }^{* *} \mathrm{P}<0.001 \mathrm{vs}$. the control, ${ }^{*} \mathrm{P}<0.05$, ${ }^{\# \#} \mathrm{P}<0.001$ vs. co-treatment (Combi)].

with gefitinib and vorinostat in both PC9 and PC9GR cells compared with that of vorinostat alone (Fig. 4A). To verify the contribution of ROS in combined treatment-induced
HSP90 cleavage, we examined the effect of the NADPH oxidase inhibitor, AEBSF. The addition of AEBSF effectively blocked the decrease in the levels of phosphorylated and total 
D

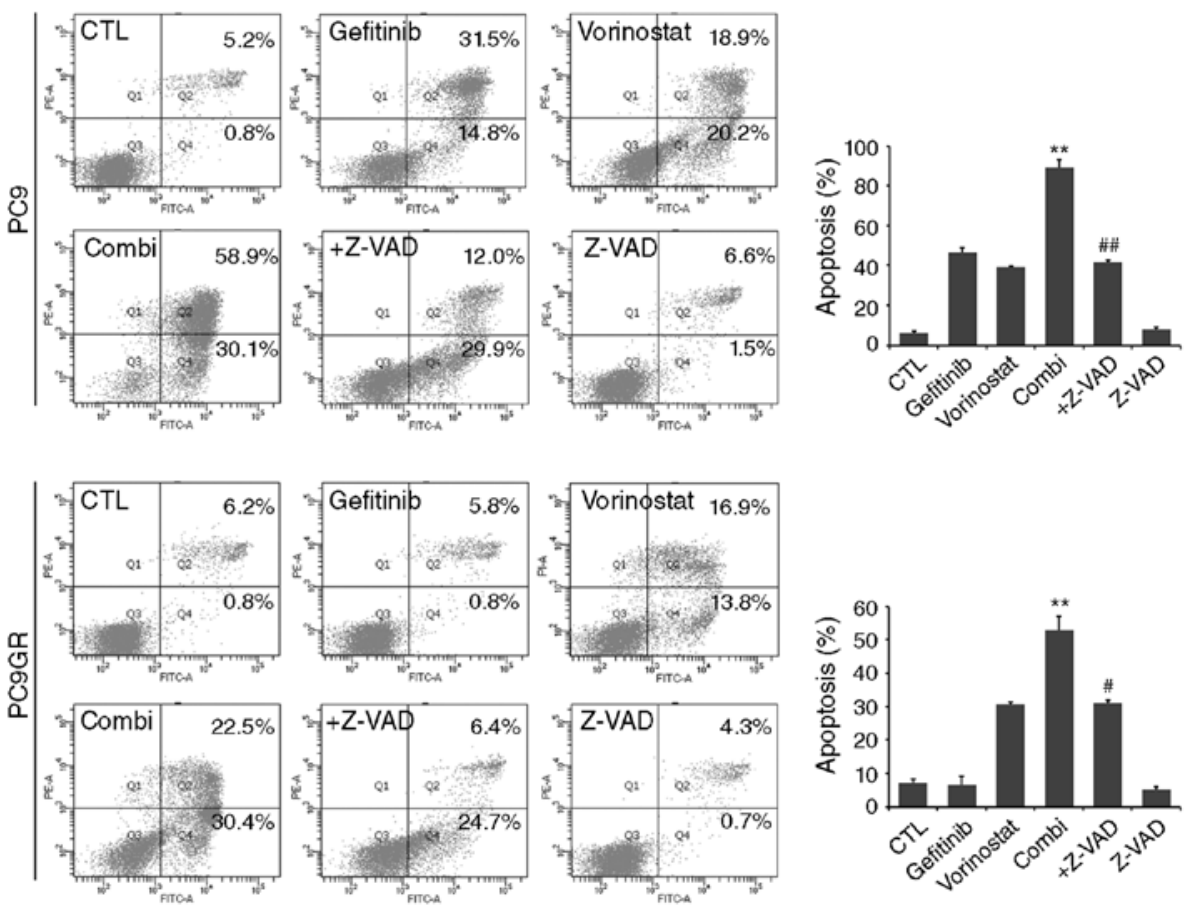

Figure 2. Continued. (D) Representative flow cytometry scatter plots of propidium iodide (PI) (y-axis) vs Annexin V-FITC (x-axis). Bar charts show quantitative data of the average of 3 independent flow cytometry experiments in PC9 and PC9GR cells ( $\mathrm{n}=3 ;{ }^{* * *} \mathrm{P}<0.001$ vs. the control, ${ }^{\sharp} \mathrm{P}<0.05,{ }^{\# \#} \mathrm{P}<0.001 \mathrm{vs}$. Combi).

A

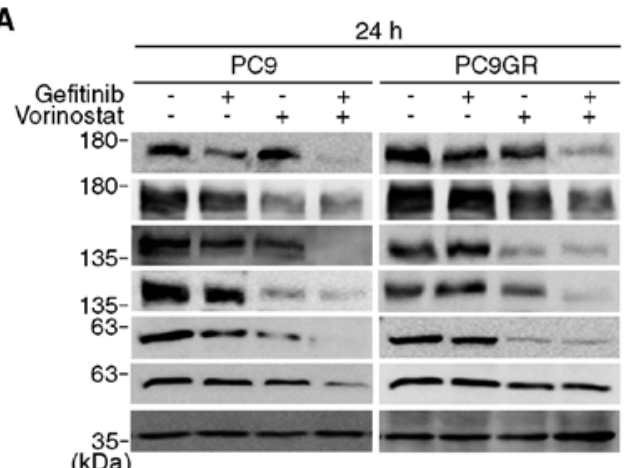

$(\mathrm{kDa})$

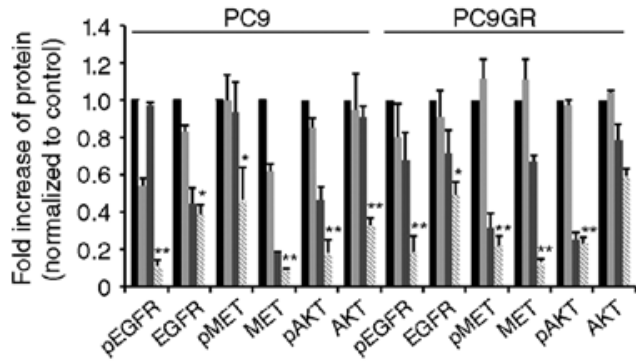

B

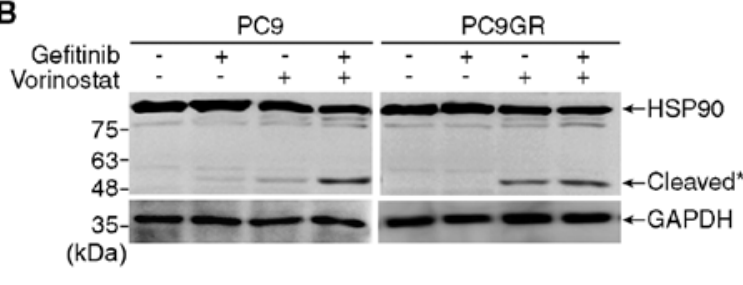

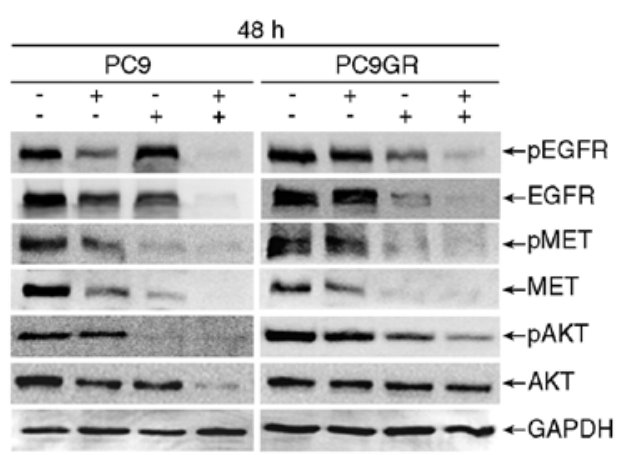
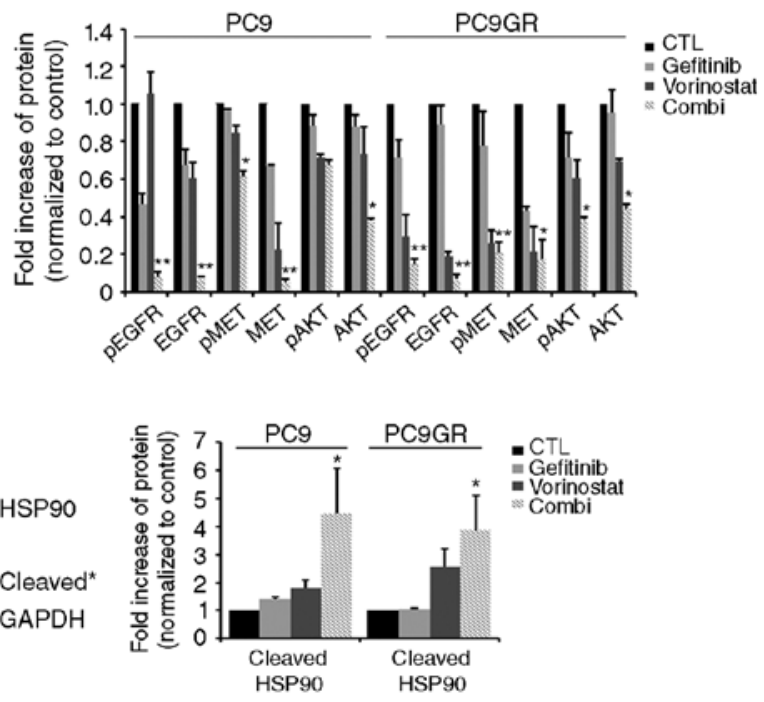

Figure 3. Co-treatment with gefitinib and vorinostat synergistically induces HSP90 cleavage and degrades its client proteins. (A) PC9 and PC9GR cells were treated $0.01 \mu \mathrm{M}$ gefitinib and/or $5 \mu \mathrm{M}$ vorinostat for 24 or $48 \mathrm{~h}$ and the levels of total or phosphorylated EGFR, MET and AKT were detected using western blotting ( $\mathrm{n}=3,{ }^{*} \mathrm{P}<0.05,{ }^{* *} \mathrm{P}<0.001$ vs. the control). (B) Cells were treated with $0.01 \mu \mathrm{M}$ gefitinib and/or $5 \mu \mathrm{M}$ vorinostat for $24 \mathrm{~h}$, and HSP90 cleavage was determined by western blotting. GAPDH was used as a loading control. Cleavage products of HSP90 are labeled with an asterisk ( $\mathrm{n}=3$, ${ }^{\mathrm{P}}<0.05 \mathrm{vs}$. the control). 
A

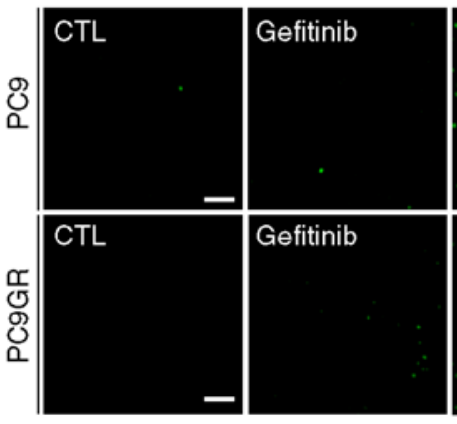

B
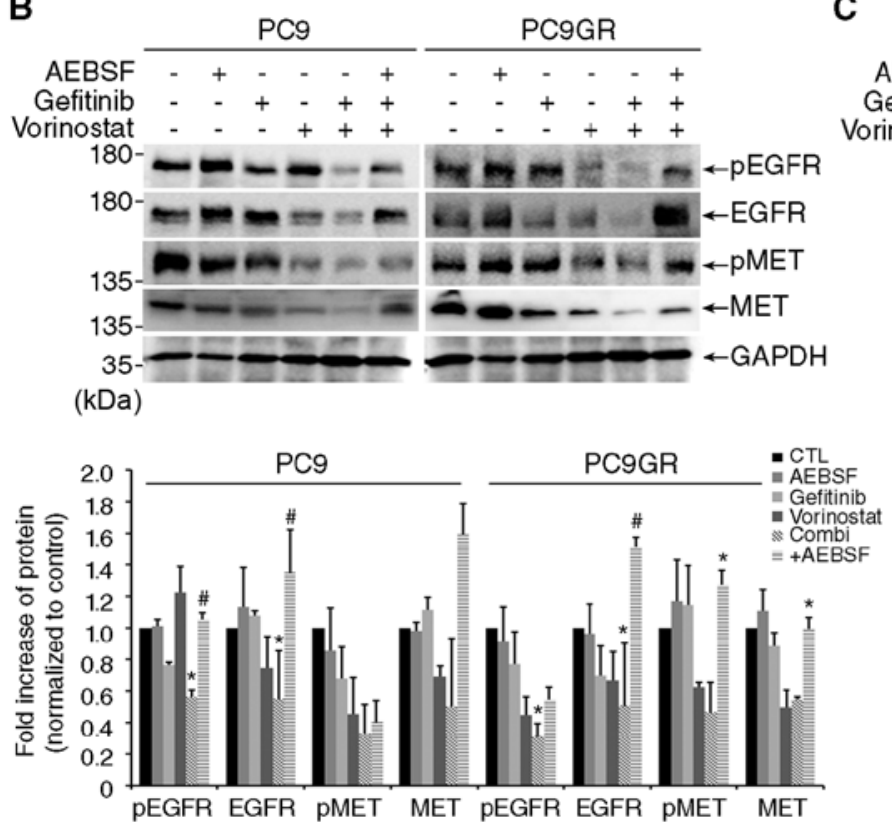

C
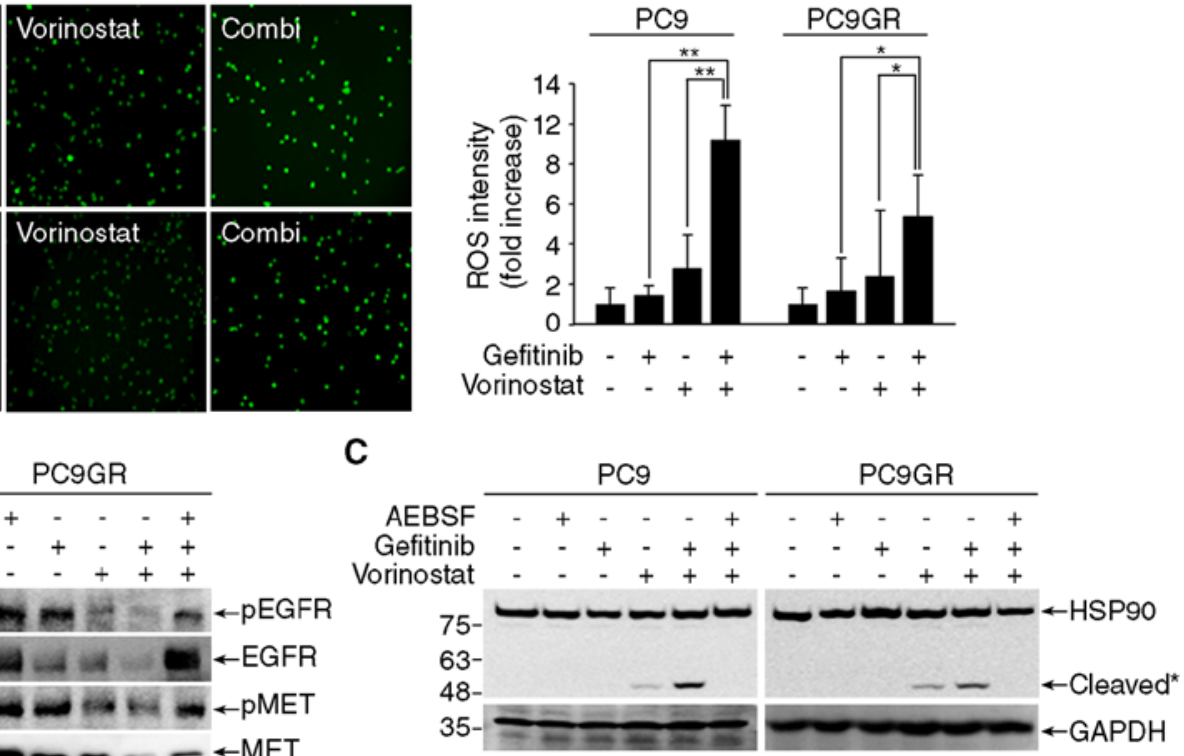

$(\mathrm{kDa})$

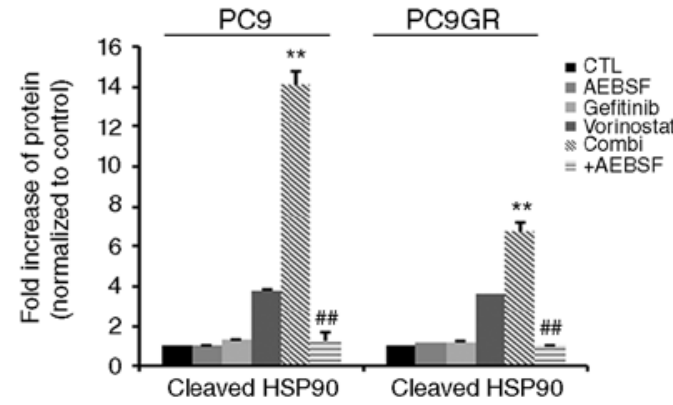

Figure 4. Co-treatment accumulates reactive oxygen species (ROS)-dependent HSP90 cleavage and client degradation. (A) PC9 and PC9GR cells were treated with $0.01 \mu \mathrm{M}$ gefitinib and/or $5 \mu \mathrm{M}$ vorinostat for $16 \mathrm{~h}$, stained with $10 \mu \mathrm{M} \mathrm{H}_{2} \mathrm{DCF}-\mathrm{DA}$, and observed by fluorescence microscopy. Scale bar, $50 \mu \mathrm{m}$. The bar graphs represent the normalized fluorescent intensity of ROS (mean $\pm \mathrm{SEM}, \mathrm{n}=3 ;{ }^{*} \mathrm{P}<0.05$, ${ }^{* *} \mathrm{P}<0.001$ ). (B and C) Cells were treated with $0.01 \mu \mathrm{M}$ gefitinib and/or $5 \mu \mathrm{M}$ vorinostat for $24 \mathrm{~h}$ with or without $100 \mu \mathrm{M} 4$-(2-aminoethyl)-benzenesulfonyl fluoride hydrochloride (AEBSF), and the level of total or phosphorylated EGFR and MET (B) and cleaved HSP90 (C) were determined by western blotting. GAPDH was used as a loading control. Cleavage products of HSP90 are labeled with an asterisk $\left[\mathrm{n}=3,{ }^{*} \mathrm{P}<0.05,{ }^{* *} \mathrm{P}<0.001\right.$ vs. the control, ${ }^{\#} \mathrm{P}<0.05,{ }^{\# \#} \mathrm{P}<0.001$ vs. co-treatment $\left.(\mathrm{Combi})\right]$.

EGFR and MET (Fig. 4B) and HSP90 cleavage (Fig. 4C) by the combined treatment. These results indicate that HSP90 cleavage induced by co-treatment with gefitinib and vorinostat is mediated by ROS production, and that these events increase the degradation of HSP90 client proteins, such as EGFR, MET and AKT.

ROS-dependent caspase activation is responsible for HSP9O cleavage by combined treatment. As ROS- or caspasemediated HSP90 cleavage is implicated in apoptosis $(13,15)$, we investigated the types of caspases that are involved in ROS-dependent cleavage of HSP90 induced by co-treatment with gefitinib and vorinostat. Addition of AEBSF inhibited the cleavage of an effector caspase- 3 and its activators, caspase- 8 and caspase-9 in PC9 and PC9GR cells exposed to the combined treatment (Fig. 5A). In accordance with these results, the caspase- 3 inhibitor Z-DEVD completely abolished HSP90 cleavage, and the inhibitors against each caspase, Z-IETD for caspase-8 and Z-LEHD for caspase-9, were partially effective in blocking HSP90 cleavage augmented by the combined treatment (Fig. 5B and C). In addition, the pan-caspase inhibitor Z-VAD reversed the degradation of HSP90 clients and HSP90 cleavage as well as apoptosis in both cell lines co-treated with gefitinib and vorinostat (Figs. 5D and 2C). Moreover, the addition of AEBSF attenuated cell death induced by the combined treatment in the PC9 and PC9GR cells (Fig. 5E). These results indicate that HSP90 cleavage by co-treatment with gefitinib and vorinostat is mediated by ROS-dependent caspase activation, and these events increase apoptosis by degrading HSP90 client proteins, such as EGFR, MET and AKT.

\section{Discussion}

The present study revealed that vorinostat in combination with gefitinib exerted a synergistic anticancer effect on NSCLC PC9 and PC9GR cells. Our results indicate that ROS-dependent caspase activation by co-treatment with gefitinib and vorinostat mediated HSP90 cleavage, and that these events increased apoptosis by degrading HSP90 client proteins, such as EGFR, 
A
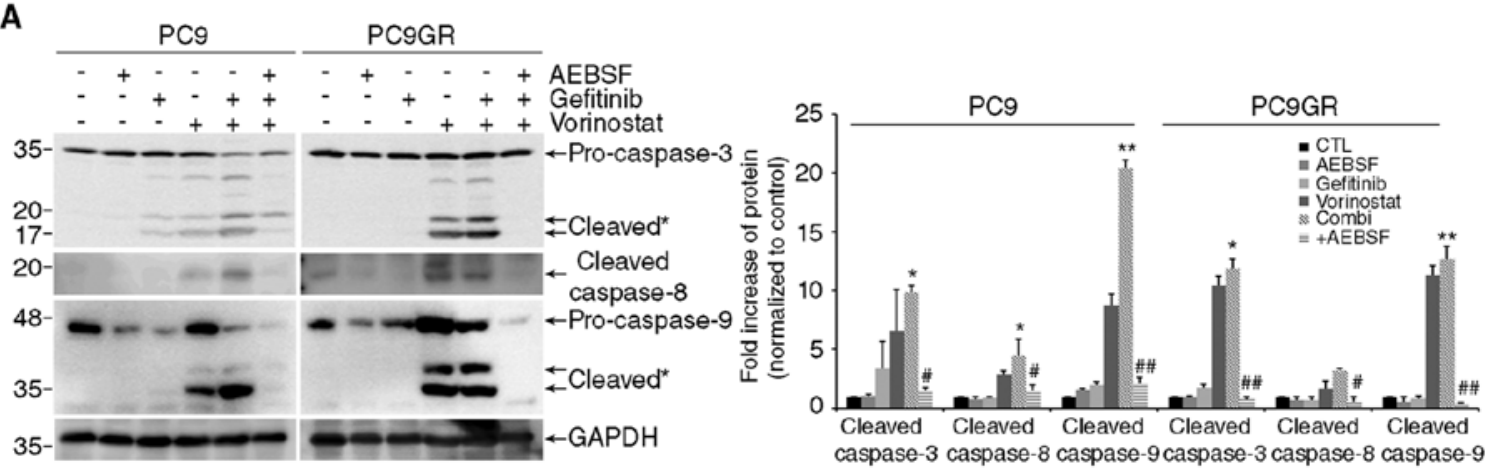

$(\mathrm{kDa})$
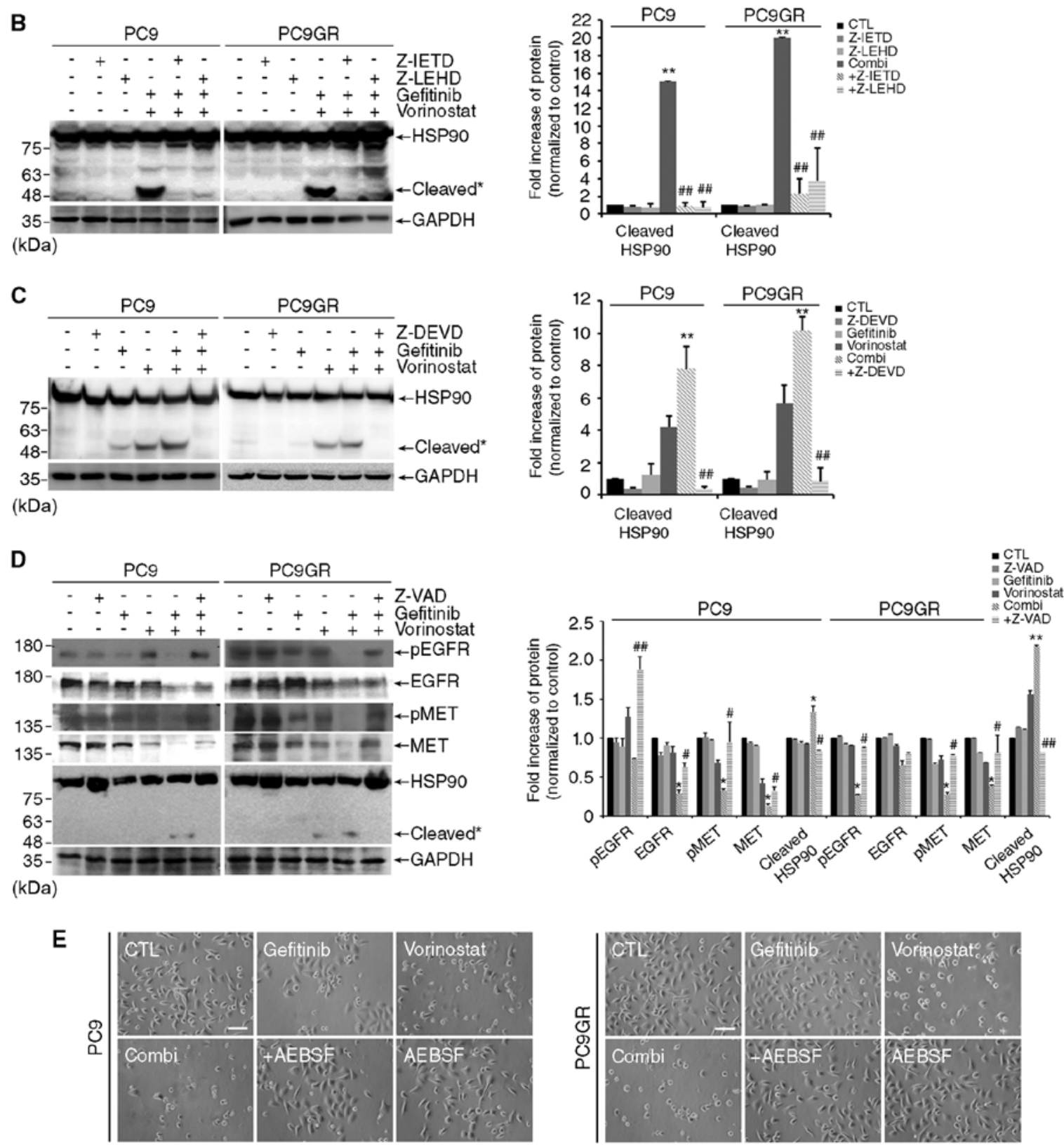

Figure 5. Co-treatment with gefitinib and vorinostat induces HSP90 cleavage through reactive oxygen species (ROS)-dependent caspase activation. (A) PC9 and PC9GR cells were treated with $0.01 \mu \mathrm{M}$ gefitinib and/or $5 \mu \mathrm{M}$ vorinostat for $48 \mathrm{~h}$ with or without $100 \mu \mathrm{M}$ AEBSF, and caspase-3, -8 , and -9 cleavage was determined by western blotting. Cleavage products of caspase $-3,-8$, and -9 are labeled with an asterisk $\left[\mathrm{n}=3,{ }^{*} \mathrm{P}<0.05\right.$, ${ }^{* *} \mathrm{P}<0.001 \mathrm{vs}$. the control, ${ }^{*} \mathrm{P}<0.05$, ${ }^{\# \#} \mathrm{P}<0.001$ vs. co-treatment (Combi)]. (B and C) Cells were treated with $0.01 \mu \mathrm{M}$ gefitinib and/or $5 \mu \mathrm{M}$ vorinostat for $24 \mathrm{~h}$ with or without $50 \mu \mathrm{M} Z$-IETD-FMK, Z-LEHD-FMK (B), or Z-DEVD-FMK (C), and HSP90 cleavage was determined by western blotting ( $\mathrm{n}=3,{ }^{* *} \mathrm{P}<0.001 \mathrm{vs}$. the control, ${ }^{\# \#} \mathrm{P}<0.001 \mathrm{vs}$. Combi). (D) Cells were treated with $0.01 \mu \mathrm{M}$ gefitinib and/or $5 \mu \mathrm{M}$ vorinostat for $24 \mathrm{~h}$ with or without $50 \mu \mathrm{M}$ Z-VAD-FMK. The levels of total or phosphorylated EGFR and MET and HSP90 cleavage were detected by western blotting. GAPDH was used as a loading control. Cleavage products of HSP90 are labeled with an asterisk ( $\mathrm{n}=3,{ }^{*} \mathrm{P}<0.05,{ }^{* *} \mathrm{P}<0.001$ vs. the control, ${ }^{\#} \mathrm{P}<0.05,{ }^{\# \#} \mathrm{P}<0.001$ vs. Combi). (E) Cells were treated with $0.01 \mu \mathrm{M}$ gefitinib and/or $5 \mu \mathrm{M}$ vorinostat with or without $100 \mu \mathrm{M}$ AEBSF for $48 \mathrm{~h}$ and cell death was assessed. 
MET and AKT. Accordingly, we suggest fundamental evidence to support that a combination of HDACI and gefitinib can be used to overcome gefitinib resistance in NSCLC.

Targeted anticancer therapies have appreciably advanced since the last decade. In 2015, gefitinib, a non-covalent inhibitor, was approved by the U.S. Food and Drug Administration for the first-line treatment of metastatic lung cancer with an EGFR-activating mutation, such as an EGFR exon 19 deletion and an EGFR L858R mutation in exon 21 found in $80-90 \%$ of all EGFR mutations in NSCLC (16). However, progression-free survival of this therapy was reported to be rather disappointing due to the development of diverse resistance (17). One of the main mechanisms by which resistance to gefitinib is acquired is the EGFR T790M mutation. Another resistance mechanism is activation or upregulation of bypass RTKs (MET, ERBb3, AXL and IGF1R) and their downstream signaling molecules (PI3K, AKT, MEK and JAK) $(17,18)$. Although the second- and third-generation covalent inhibitors against EGFR T790M have been used for NSCLC, resistance of these drugs has occurred in patients with new resistant EGFR mutations and bypass pathways. To overcome resistance against EGFR-TKIs, therefore, it is useful to downregulate RTKs, for example EGFR itself or MET, which takes charge of bypassing signaling pathways, or a downstream signaling molecule, AKT. Our results showed that HDACI can reduce the level of EGFR, MET and AKT which are responsible for gefitinib resistance. These results associated with overcoming gefitinib-resistance will expand the opportunity to develop combination therapies with EGFR-TKIs as an alternative for prolonging the control of the disease.

HDACIs, a group of epigenetic drugs, have been evaluated for their synergistic effects when combined with various conventional chemotherapeutic or targeted cancer therapies for different types of tumors $(19,20)$. It has been reported that HDACIs can increase sensitivity and reverse resistance to EGFR-TKIs in lung cancers. The suggested mechanisms of synergy are the induction of E-cadherin, BIM and $\operatorname{BAX}(5,21,22,30)$, inhibition of the IGF 1R-AKT pathway (23), and accumulation of ROS by upregulating the major mitochondrial porin voltage-dependent anion-selective channel protein 1 and modulating the $\mathrm{c}-\mathrm{Myc} / \mathrm{NRF} / \mathrm{KEAP} 1$ pathway (24) in NSCLC cell lines. It has also been reported that an HDACI potentiates apoptosis induced by an EGFR-TKI by HSP90 acetylation-dependent depletion of key survival signaling proteins, including AKT, EGFR, c-Src and STAT3 (2). Acetylation of K294 (HSP90a)/K287 (HSP90ß) reduces its affinity to the co-chaperone machinery, leading to the degradation of client proteins and apoptosis $(25,26)$. Therefore, deacetylation of these proteins by HDAC6 stabilizes HSP90 function, and the HDACI induces apoptosis through acetylation and activation of HSP90 in cancer $(3,25,27)$. One novel finding of our study was that co-treatment with gefitinib and vorinostat had an additional mechanism of caspase-mediated degradation of HSP90 with a molecular weight of $\sim 55 \mathrm{kDa}$ in PC9 and PC9GR NSCLC cells. Moreover, HDACI-induced cleavage of HSP90 was also detected in other types of cancer cells [renal (SN12C, ACHN and 786O), prostate (PC3), and breast (MCF-7 and MDA-MB-231) cancer cell lines (data not shown)]. HSP90 cleavage attenuates the function of HSP90, results in destabilization of client proteins, and potentiates apoptosis in cancer cells. There have been reports showing that HDACI activates ROS-dependent caspase activation, triggering extrinsic or intrinsic apoptosis in various types of cancer cells $(28,29)$, and that oxidative stress and caspase-10 induces HSP90 cleavage $(13,15)$. Therefore, we predicted that vorinostat would induce HSP90 cleavage through ROS-dependent caspase activation, and our results have supported this prediction.

Notably, vorinostat was shown to overcome EGFR-TKI resistance and synergize with EGFR-TKIs in NSCLCs in vivo. Busser et al and Jeannot et al reported that the combination of gefitinib and vorinostat inhibited tumor growth in a gefitinib-resistant $\mathrm{H} 358$ xenograft model by activating BAX released from acetylated Ku70 and inhibiting the IGF 1R-AKT pathway, respectively $(22,23)$. Nakagawa et al showed that the combined use of vorinostat and EGFR-TKI (gefitinib or osimertinib) synergistically regressed tumors in xenograft models using PC9 and PC9GR (T790M) with BIM deletion polymorphism through re-expression of active BIM (21). In addition, a phase I/II clinical study of co-treatment with gefitinib and vorinostat showed a potential improvement in patients with advanced NSCLC (31). In our study, we found in vitro that co-treatment with gefitinib and vorinostat presented a new mechanism by which to induce cell death in NSCLC, although we did not perform in vivo experiments. We showed that activation of ROS-dependent caspases led to the downregulation of HSP90 client proteins through HSP90 cleavage in PC9 and PC9GR cells. Thus, current research has led to the presumption that HDACIs are promising drugs that potentiate the therapeutic efficacy of EGFR-TKIs. Our results, which present the synergistic antitumor activity of vorinostat in combination with gefitinib, provide the supportive rationale for the future design of clinical trials based on these inhibitors in NSCLC patients.

\section{Acknowledgements}

Not applicable.

\section{Funding}

This study was supported by grants from the Korean Health Technology R\&D Project, Ministry of Health and Welfare, Republic of Korea (HI15C0972), and from the Asan Institute for Life Sciences, Asan Medical Center (2018-450) Seoul, Republic of Korea.

\section{Availability of data and materials}

The analyzed data sets generated during the study are available upon reasonable request from the corresponding author.

\section{Authors' contributions}

SEP designed and wrote the manuscript. DEK, JSL and MJK conducted the research. JKR provided the cell lines and analyzed the results. SYJ and EKC helped to collect the data and analyze the results. CSK and JJH were the leading principal investigators who directed the study, were involved in the conception of the study, and drafted the manuscript. All authors read, revised and approved the manuscript and agree 
to be accountable for all aspects of the research in ensuring that the accuracy or integrity of any part of the study were appropriately investigated and resolved.

\section{Ethics approval and consent to participate}

Not applicable.

\section{Patient consent for publication}

Not applicable.

\section{Competing interests}

The authors declare that they have no competing interests.

\section{References}

1. Beere HM: 'The stress of dying': The role of heat shock proteins in the regulation of apoptosis. J Cell Sci 117: 2641-2651, 2004

2. Edwards A, Li J, Atadja P, Bhalla K and Haura EB: Effect of the histone deacetylase inhibitor LBH589 against epidermal growth factor receptor-dependent human lung cancer cells. Mol Cancer Ther 6: 2515-2524, 2007.

3. Socinski MA, Goldman J, El-Hariry I, Koczywas M, Vukovic V, Horn L, Paschold E, Salgia R, West H, Sequist LV, et al: A multicenter phase II study of ganetespib monotherapy in patients with genotypically defined advanced non-small cell lung cancer Clin Cancer Res 19: 3068-3077, 2013.

4. Jhaveri K, Taldone T, Modi S and Chiosis G: Advances in the clinical development of heat shock protein $90(\mathrm{Hsp} 90)$ inhibitors in cancers. Biochim Biophys Acta 1823: 742-755, 2012.

5. Witta SE, Gemmill RM, Hirsch FR, Coldren CD, Hedman K, Ravdel L, Helfrich B, Dziadziuszko R, Chan DC, Sugita M, et al Restoring E-cadherin expression increases sensitivity to epidermal growth factor receptor inhibitors in lung cancer cell lines. Cancer Res 66: 944-950, 2006.

6. Neal JW and Sequist LV: Complex role of histone deacetylase inhibitors in the treatment of non-small-cell lung cancer. J Clin Oncol 30: 2280-2282, 2012.

7. Hoang T, Campbell TC, Zhang C, Kim K, Kolesar JM, Oettel KR, Blank JH, Robinson EG, Ahuja HG, Kirschling RJ, et al: Vorinostat and bortezomib as third-line therapy in patients with advanced non-small cell lung cancer: A wisconsin oncology network phase II study. Invest New Drugs 32: 195-199, 2014

8. Rho JK, Choi YJ, Lee JK, Ryoo BY, Na II, Yang SH, Lee SS, Kim CH, Yoo YD and Lee JC: The role of MET activation in determining the sensitivity to epidermal growth factor receptor tyrosine kinase inhibitors. Mol Cancer Res 7: 1736-1743, 2009.

9. Rho JK,Lee IY, Choi YJ, Choi CM, Hur JY, Koh JS, Lee J, Suh BC Song HJ, Salgaonkar P, et al: Superior efficacy and selectivity of novel small-molecule kinase inhibitors of T790M-mutant EGFR in preclinical models of lung cancer. Cancer Res 77: 1200-1211, 2017.

10. Gazdar AF: Epidermal growth factor receptor inhibition in lung cancer: The evolving role of individualized therapy. Cancer Metastasis Rev 29: 37-48, 2010.

11. Sadiq AA and Salgia R: MET as a possible target for non-small-cell lung cancer. J Clin Oncol 31: 1089-1096, 2013.

12. Mahalingam D, Swords R, Carew JS, Nawrocki ST, Bhalla K and Giles FJ: Targeting HSP90 for cancer therapy. Br J Cancer 100: $1523-1529,2009$.

13. Beck R, Verrax J, Gonze T, Zappone M, Pedrosa RC, Taper H, Feron $\mathrm{O}$ and Calderon PB: Hsp90 cleavage by an oxidative stress leads to its client proteins degradation and cancer cell death. Biochem Pharmacol 77: 375-383, 2009.
14. Pantano C, Shrivastava P, McElhinney B and Janssen-Heininger Y: Hydrogen peroxide signaling through tumor necrosis factor receptor 1 leads to selective activation of c-Jun $\mathrm{N}$-terminal kinase. J Biol Chem 278: 44091-44096, 2003.

15. Chen H, Xia Y, Fang D, Hawke D and Lu Z: Caspase-10-mediated heat shock protein 90 beta cleavage promotes UVB irradiationinduced cell apoptosis. Mol Cell Biol 29: 3657-3664, 2009.

16. Jackson SE and Chester JD: Personalised cancer medicine. Int J Cancer 137: 262-266, 2015.

17. Chong CR and Jänne PA: The quest to overcome resistance to EGFR-targeted therapies in cancer. Nat Med 19: 1389-1400, 2013.

18. Wheeler DL, Dunn EF and Harari PM: Understanding resistance to EGFR inhibitors-impact on future treatment strategies. Nat Rev Clin Oncol 7: 493-507, 2010.

19. Bose P, Dai Y and Grant S: Histone deacetylase inhibitor (HDACI) mechanisms of action: Emerging insights. Pharmacol Ther 143: 323-336, 2014

20. Minucci S and Pelicci PG: Histone deacetylase inhibitors and the promise of epigenetic (and more) treatments for cancer. Nat Rev Cancer 6: 38-51, 2006 .

21. Nakagawa T, Takeuchi S, Yamada T, Ebi H, Sano T, Nanjo S, Ishikawa D, Sato M, Hasegawa Y, Sekido Y and Yano S: EGFR-TKI resistance due to BIM polymorphism can be circumvented in combination with HDAC inhibition. Cancer Res 73: 2428-2434, 2013.

22. Busser B, Sancey L, Josserand V, Niang C, Khochbin S, Favrot MC, Coll JL and Hurbin A: Amphiregulin promotes resistance to gefitinib in nonsmall cell lung cancer cells by regulating Ku70 acetylation. Mol Ther 18: 536-543, 2010.

23. Jeannot V, Busser B, Vanwonterghem L, Michallet S, Ferroudj S, Cokol M, Coll JL, Ozturk M and Hurbin A: Synergistic activity of vorinostat combined with gefitinib but not with sorafenib in mutant KRAS human non-small cell lung cancers and hepatocarcinoma. Onco Targets Ther 9: 6843-6855, 2016.

24. Leone A, Roca MS, Ciardiello C, Terranova-Barberio M, Vitagliano C, Ciliberto G, Mancini R, Di Gennaro E, Bruzzese F and Budillon A: Vorinostat synergizes with EGFR inhibitors in NSCLC cells by increasing ROS via up-regulation of the major mitochondrial porin VDAC1 and modulation of the c-Myc-NRF2KEAP1 pathway. Free Radic Biol Med 89: 287-299, 2015.

25. Bali P, Pranpat M, Bradner J, Balasis M, Fiskus W, Guo F, Rocha K, Kumaraswamy S, Boyapalle S, Atadja P, et al: Inhibition of histone deacetylase 6 acetylates and disrupts the chaperone function of heat shock protein 90: A novel basis for antileukemia activity of histone deacetylase inhibitors. J Biol Chem 280: 26729-26734, 2005.

26. Scroggins BT, Robzyk K, Wang D, Marcu MG, Tsutsumi S, Beebe K, Cotter RJ, Felts S, Toft D, Karnitz L, et al: An acetylation site in the middle domain of Hsp90 regulates chaperone function. Mol Cell 25: 151-159, 2007.

27. Nishioka C, Ikezoe T, Yang J, Takeuchi S, Koeffler HP and Yokoyama A: MS-275, a novel histone deacetylase inhibitor with selectivity against HDAC1, induces degradation of FLT3 via inhibition of chaperone function of heat shock protein 90 in AML cells. Leuk Res 32: 1382-1392, 2008.

28. Ruefli AA, Ausserlechner MJ, Bernhard D, Sutton VR, Tainton KM, Kofler R, Smyth MJ and Johnstone RW: The histone deacetylase inhibitor and chemotherapeutic agent suberoylanilide hydroxamic acid (SAHA) induces a cell-death pathway characterized by cleavage of Bid and production of reactive oxygen species. Proc Natl Acad Sci USA 98: 10833-10838, 2001.

29. Kim DH, Lee J, Kim KN, Kim HJ, Jeung HC, Chung HC and Kwon HJ: Anti-tumor activity of N-hydroxy-7-(2-naphthylthio) heptanomide, a novel histone deacetylase inhibitor. Biochem Biophys Res Commun 356: 233-238, 2007.

30. Ng KP, Hillmer AM, Chuah CT, Juan WC, Ko TK, Teo AS, Ariyaratne PN, Takahashi N, Sawada K, Fei Y, et al: A common BIM deletion polymorphism mediates intrinsic resistance and inferior responses to tyrosine kinase inhibitors in cancer. Nat Med 18: 521-528, 2012.

31. Han JY, Lee SH, Lee GK, Yun T, Lee YJ, Hwang KH, Kim JY and Kim HT: Phase I/II study of gefitinib (Iressa $\left({ }^{\circledR}\right)$ ) and vorinostat (IVORI) in previously treated patients with advanced non-small cell lung cancer. Cancer Chemother Pharmacol 75: 475-483, 2015. 